\title{
Concerning the Origin of the Spacetime with Regard to a Multi-rest States Physical Structure
}

\author{
Ahmed Isam \\ Independent Researcher \\ Khartoum, Sudan. E-mail: ahmedisam777@gmail.com
}

\begin{abstract}
In this paper, we investigate the ontological hypothesis, which implies that the spacetime is not the ultimate structure in our universe, and its existence emerges from a deeper physical entity. By using a very simple approach based on a classical problem, regarding the propagation of electromagnetic waves in empty vacuum. We were able to deduce that this deeper entity is just an omnipresent multi-rest states physical structure; aether. After that, we try to see how this aether fits in the universe that we exist in. The outcome is that its existence causes the emergence of some basic phenomena that our universe is built on. At the microscopic scale it turned out to be a source of the essential quantum phenomenon, which is currently known as the wave - particle duality. On the other hand, at the macroscopic scale it causes the emergence of spacetime curvature, around huge, massive objects like Earth, and finally, we try to visualize the prospects of aether, and how it can be a promising alternative for the dark matter.
\end{abstract}

Keywords: Emergent Spacetime ; Origin of Relativity ; Quantum theory ; Dark Matter

\section{1- Introduction:}

Consider a liquid water exist within a pool as an example. You can realize that at the macroscopic scale, this water has physical properties, e.g. surface tension, which its basic microscopic constituent element ( water molecule $\mathrm{H}_{2} \mathrm{O}$ ) does not have. Descriptively, this can be called emergence, which in general reflects the collective behavior of a large number of microscopic ( deeper) elements, that individually, each microscopic element has different physical properties in comparison with the structure, which these elements generate at the macroscopic level . The generated macroscopic entity can be called an emergent structure. From here, regarding our subject in this paper, we can propose a simple question; is spacetime an emergent structure? Or in other words; does spacetime rise from something deeper that has different properties compared with its own? This might indicate that both space and time are just notions appear at the observable macroscopic scale, and they will cease to exist below certain minimum limits; Planck length, and Planck time respectively. At these crucial minimum limits, some sort of a deep physical structure or mechanism, can reveal itself, and it might not be spatial or temporal at all, and yet, it generates the spacetime at a larger observable scale. This notion resembles the previous example: within a water pool, a single molecule of $\mathrm{H}_{2} \mathrm{O}$ does not have the physical properties of the liquid water, and yet, it generates it at a larger scale due to collective behavior of large number of the same molecules.. Form a vigorous theoretical research, many physicists have reached to the conclusion that in order to merge the gravitational force within the quantum realm, the 
concept of the spacetime at the very microscopic scale $\left(\approx 10^{-35} \mathrm{~m}\right)$ should be altered or changed. The theories which address the emergence of the spacetime are generally advocated within the discipline of quantum gravity [1-3]. Although the theoretical work on these theories is still on progress, simple basic notions regarding them can be considered. For example, Loop Quantum Gravity [4] proposes the classical space which we macroscopically observe, should emerge from a microscopic structure called a spin network. It contains specific elements called nodes and links. They are related to the elementary quanta of volume and area respectively. On the other hand, its major competitor, string theory introduces a different picture. It proposes that the elementary constituent of our physical fields is a vibrating strand (loop) of energy, called a string. The gravitational field is composed of a huge number of strings; each one of them is called a graviton. Relevantly, general relativity describes the gravitational field as a distorted state of spacetime. By merging the two concepts together, the notion of space and time in string theory - within specific limits described by the theory - emerges from a tremendous number of gravitons that their excitations are in a coherent state; vibrate in a similar, organized pattern [5].

But the problem with all these different paradigms about how space emerges from a deeper reality is that they are beyond our observational (experimental) reach. Because quantum gravity theories work on a very small scale related to the value of Planck length $\left(\approx 10^{-35} \mathrm{~m}\right)$. This by itself enlightens us about the great difficulty to obtain any kind of direct evidence that can really tell us what is really going down there. But for now, and to a "very limited" extent, this obstacle can be defied by using an opposite perspective, which implies that the large macroscopic scale of spacetime may have observable consequences (clues) from the obscure ( unknown) microscopic properties of itself. This by itself entails that we should carefully probe all the observable macroscopic properties of the spacetime, even the intuitive one, because they may contain - hidden or missed - clues about its hidden microscopic structure. Recently, we have used this approach, and we have reached to the conclusion that both space and time have discrete (discontinuous) structure at the microscopic scale, in a similar way - but not necessary the same - as proposed by loop quantum gravity. The consequent macroscopic observations for this discreteness are the existence of the opposite spatial directions; origin position, with respect to the discreteness of space, and the flow of time from the present toward the future regarding the discreteness of time [6].

Regarding the problem which is concerned with the hidden structure that causes the emergence of the spacetime, I think the macroscopic property that we need to probe is the constancy of the speed at which light propagates. This speed is the physical constant that determines how both space and time should be curved or distorted with motion and around massive objects ( gravitational fields) [7]. Which by itself might indicate a strong relationship between the constancy of this speed, and the obscure structure; mechanism, that causes the emergence of the spacetime. Therefore, in order to produce an objective discussion, let us propose a simple question; why does light propagate with a constant speed? Or with different words why does the speed of light represent a physical constant? Is it really a fundamental principle as we currently assume or just consequence for another deeper mechanism? At first glance, this kind of questions might seem it will lead us to a philosophical debate rather than a scientific one. But if we make a historical gesture, we can see that questions like these usually had opened the door for a deeper level of understanding our physical reality. For example, in 1900s, the French physicist Louis de Broglie had wondered "why" the electron follows only certain orbits around the nucleus in Bohr's theory [8] of the hydrogen atom ; he did not deal with it as a fundamental principle, but rather as a consequence for some deeper reality. This in turn, led him to propose the existence of matter waves in 1924 in order to justify Bohr's notion [9-10].

For the sake of objectivity in this section, we shall only deal with the wave nature of light, and leave its particle properties to section 3, where it will be considered in details. The speed at which light propagates was calculated theoretically for the first time in 1864, by the British physicist James Clerk Maxwell. He predicted that an accelerated electric charge can generate linked electric and magnetic disturbances that can propagate through the space as wave. This led to the conclusion that light itself is a type of Electromagnetic (EM) waves, which its speed can be obtained from his formula [11]: 


$$
c=\frac{1}{\sqrt{\epsilon_{0} \mu_{0}}} \approx 3 \times 10^{8} \mathrm{~m} / \mathrm{s}
$$

Thus, the speed of light depends on two constant values that appear in the right side this equation, which are $\left(\epsilon_{0}\right)$ and $\left(\mu_{0}\right)$. They are defined as electric permittivity and magnetic permeability of the free space. This creates a theoretical problem which comes from the previous definitions. Because they imply that the space has a property to convey or transmit EM fields, but the question is; what is exactly this property? Based on a pure classical ground, the importance of this property signifies even more, when the values of these EM fields change with time in a specific point in space and propagates as EM waves. At this situation, this "hidden" property of space will be responsible for conveying or transmitting these waves from one place to another. But again, what is it? Because space as we currently know has four values (dimensions) ; length, width, height, and a flow of time, which are measured with meter and second respectively. None of these values seem to be related to EM entity.

The objectivity of this question becomes more prominent, if we compare the previous situation with the gravitational interactions. Today, we assume that space has the property to covey or transmit the gravitational field that originates from a missive object like the Sun. Even more, these fields can propagate as Gravitational Waves (GW), and we can simply say that the space or vacuum has the ability to convey or transmit these waves. But if we compare these phenomena with EM phenomena, we can see a big difference. According to Einstein's General Theory of Relativity (GTR), the gravitational field around a massive object is not merely a field passes through space; it is a state or distortion in the space itself. The vacuum has the property (ability) to convey or transmit the GW, because simply these waves are propagating disturbances (distortions) in its own structure. Therefore, the property that allow vacuum to transmit GW is identified and known for us, which is its four dimensions ( $x, y, z, t)$; $G W$ are just a propagating distortions in the dimensions themselves. But regarding EM waves, the source of the property that allows vacuum to convey or transmit these waves remains unknown. Albert Einstein knew about the problem of this "hidden property" . But he chose to ignore it, in 1938, he stated that: "Our only way out seems to be to take for granted the fact that space has the physical property of transmitting electromagnetic waves, and not to bother too much about the meaning of this statement". [ 12 ]

To solve this problem, we can assume that if the four dimensions $(x, y, z, t)$ stands for the physical property that allows vacuum to transmit GW. Then, may be the vacuum has some sort of a hidden dimension; fifth dimension, that can stand for its unknown property which allows it to convey EM waves. A similar step was taken in 1919 by the German mathematician and physicist Theodor Kaluza. He hypothesized that the vacuum might have a fifth dimension that could be responsible for the EM phenomena. His goal was to merge Einstein's GTR with Maxwell's equations of EM in order to come with a unified theory. But if his work was successful, then intuitively, it would be a solution to our problem. Because this hidden ( fifth) dimension of the vacuum should stand for its unknown property that allow it to transmit EM waves. But Kaluza's original approach was obstructed by some observational discrepancies [13-14].

By using a similar approach to the problem, instead of adding a dimension to the vacuum that can justify its EM properties, we can simply add an omnipresent component ( a carrier medium) that can justify for the EM phenomena. Hence, the need for this unknown property of vacuum will simply vanish, because it will be replaced by this carrier medium. One of the first implications for such a hypothesis is that the meaning of the previous equation of Maxwell will be changed $c=1 / \sqrt{\epsilon_{0} \mu_{0}}$. As we know from classical physics, wave is defined as a propagating disturbance. The propagation of a wave is a dynamical process which its speed depends on the internal properties of its carrier medium. Take sound waves as example, its propagation speed mainly depends on the temperature and the pressure of its carrier medium (air). We can take a more accurate example which is string wave. Its speed of propagation can be obtained from a simple equation as $v=\sqrt{F L / m},[15]$ where $F$ is the tension on string, $L$ is the length of the string, and $m$ is its mass. All of the three values which determine the speed at which the wave propagates represent internal properties within its carrier medium (string). Similarly, with regard to EM waves, the 
values which determine its speed $\left(\epsilon_{0}, \mu_{0}\right)$ should stand for the internal properties of its carrier medium, which implies that their concepts should be changed to electric permittivity and magnetic permeability of the carrier medium ( ether). Although this concept was hugely endorsed by many physicists in the $19^{\text {th }}$ century [16], unfortunately, just like Kaluza's idea about the fifth dimension, it had faced a major conflict between the "proposed" theoretical models, and the observational findings. The famous one, is the huge discrepancy between the theoretical assumption of " motion with respect to ether", and the findings of Michelson-Morley's Experiment [17]. These conflicts have become even larger with the development of more abstract physical concepts that set the foundation of the Quantum Theory. All of that have led many physicists to neglect or undermine this concept.

Today, the statement which implies that light is a wave that propagates through an empty vacuum does not seem strange or odd to some people. But for the sake of honesty, I find it carries some sort of epistemological paradox, which can be initiated by the following question; how can we understand something that it does not follow a specific laws or concepts in its behavior? I think the answer is simply no. Because we usually say that we understand that something, after we had decoded the concepts (laws) that governs its behavior. But at the first place, if that something does not follow a specific set of concepts ( laws) in its behavior, then we can never understand it, because it is basically not understandable. Similarly, Physics is a branch of science that is concerned with understanding the laws (concepts) of nature, and in order to that, we have to admit that nature has viable laws (concepts) at the first place. The paradox comes when we consider the wave concept in physics, which defines it as a propagating disturbance that carry energy from one place to another. The disturbance itself can be visualized as a difference between initial and final states which are taken by the internal structure of a preexisting entity; carrier medium. Therefore, you cannot have a disturbance, without having something to be disturbed at the first place, from here we can see that a wave is a dependent physical entity; its existence depends on the presence of another underlying structure (carrier medium). This concept (law) is true for all types of waves that we observe in nature like sound waves and water wave. Two types of waves that we currently know defy this concept (law); light and matter waves. If we assume that light and matter waves are two types that do not have a carrier medium to propagate within, which means they are independent physical entities; apart from the spacetime, their existence does not depend on underlying structure. From here, if we accept this assumption, then, this clearly revokes the concept (law) of waves in physics, and the paradox becomes obvious; how can we understand nature if it does not follow a specific concept (laws) in its behavior? This in turn, leads us back to the question that I have asked at the beginning of this paragraph. Because at this situation - since nature does not have viable concepts - each time we address a concept or law, we have to throw a coin to see where we can apply that law (concept), and where we do not. This in turn undermines the essence of physics as available scientific discipline, since its major aim is to understand the laws (concepts) that govern the behavior of nature.

Anyone may simply argue that this is not a paradox or even a problem, because the definition of a wave as dependent physical entity cannot be considered as a general concept or law in physics. But it is just a limited concept that is restricted to mechanical waves. Therefore, the statement that implies that EM waves propagate in empty vacuum ( independent physical entity) does not make any contradiction with the definition of waves in physics, because EM is not mechanical in the first place. Hence, the contradiction simply resolves. Reservedly, I would agree with this justification. But the experimental detection of the GW in 2016[18] enforces me to reject it. Although its complex nature - A four-dimensional wave - GWs simply obey the concept of a wave in physics. They represent propagating disturbances in the structure of the spacetime itself, which entails they are dependent physical entities. They are not mechanical waves; they are field waves and the spacetime is its carrier medium. Therefore, the above justification is simply revoked. From here, I think we can conclude that all the types of waves that we observe in nature obey the general concept of a wave (independent entities), regardless of their nature, whether they are mechanical or field waves, like Sound and GW respectively. Only two types of waves are not 
included within this concept (law) : light and matter waves. Theoretically, this makes them anomalous phenomena, which consequently should lead us to question their current justification.

The contradiction signifies even more, if we take the principle of Occam's razor into account. With respect to theoretical physics, it can be stated as the following; in order to minimize the error within a theoretical justification regarding a physical phenomenon, we have to use the fewest and simplest assumptions. From here, a theory or justification can be built on one or multiple assumptions. But if they are multiple assumptions, then, these assumptions must be compatible with each other in order to produce a successful theoretical justification - just like the two postulates of special relativity. That is because if these multiple assumptions are contradictory, then this maximizes the error; a contradiction by itself can be seen as a kind of error within our theoretical understanding. For example, take the contradiction that exists between the classical GTR, and the uncertainty principle [19]. By shifting to our problem here, regarding our current justification for the existence of waves as physical phenomenon ; wave theory. We can note that this theory is built on two assumptions: the first implies that waves are dependent physical entities. And the second imposes that some of them are independent. Regarding the wave theory in physics, these two assumptions are not compatible, but on the contrary, they antagonize (contradict) each other, which indicate there is something incorrect about them. Hence, in order to minimizing the error (removing the contradiction) within the wave theory; our theoretical understanding of waves as phenomenon. According to the principle of Occam's razor ( using the fewest assumptions), since the two assumptions of the wave theory are antagonists, which means that we cannot merge them together within a single conceptual context. Then, we have to undo one of them in order to minimizing the error within the wave theory in physics.

A simple solution to this problem can be deduced from the following example; consider a person studies a hypothetical isolated system that initially has an amount of energy $20 \mathrm{~J}$. After a period of time, he /she had measured the energy of that system, and found it to be $15 \mathrm{~J}$. Then, he /she would not revoke the concept of energy conservation. Instead, he /she would assume that, either there is something wrong with the technique that he/she used to measure the energy of that system, or the initial assumption about the "isolated physical system" is simply wrong, and the system is leaking energy to the external environment by a mechanism that is unknown for him/her. I think we all agree on this is how physics works, and this makes physics a valuable science. Similarly, regarding our problem, we do not have to revoke the wave concept in physics because it does not fit with light and matter waves. Instead, I think we have to change the assumptions that we have made about light and matter waves in order to be fitted within the concept of waves. Surely, this is not the case every time. From the history of physics, we have learned that sometimes, we need to change a specific law in order to fit with an observation(s). But in this case, the old law will appear as a sort of approximation (simplification) to the new accurate law. Furthermore, the new concept may provide us with a wider perspective that can explain more phenomena, which the old one simply does not. Take for example, the insights between Newtonian Gravity (older concept), and the GTR (newer concept). But regarding our problem here (wave propagation), we can see that the situation is entirely different; all types of waves that we have discovered obey the general concept of a wave, regardless of its nature, whether it is mechanical or not, and only two types of waves antagonize this concept, which indicates there is something incorrect about the assumptions that have been made about them. In the next section, we shall consider a simple solution to this problem. But on the same time, it will change our notion regarding the fundamentality of both space and time.

\section{2- A simple mind experiment to consider:}


In section we shall consider a simple mind (hypothetical) experiment. Consider a physical system with two observers (1) and (2), located in a free vacuum. In order to avoid any unnecessary complexity, the space is considered to be classical with only three spatial dimensions $(x, y, z)$, and time $(\mathrm{t})$ is an absolute entity. This represents the space that was accepted by most physicists before the $20^{\text {th }}$ century, and it is a good approximation for our physical spactime at very low speeds $\ll c$. Obviousely, this space does not exist in the real universe, and this why I choose to call it a mind experiment; it can only be performed in our minds. But as we will be seen, this will not affect its physical validity. The experiment is consisted of two subsequent phases.

\section{1-2- Phase 1:}

Initially, the two observes are in rest with respect to each other. Each one has his own clock, and a measurement rod, with a length $(L)$, directed along the $(x)$ axis. After that, we shall apply a force in $(x)$ direction to obs(2), for a specific time interval in order to reach him a speed of $60 \mathrm{~km} / \mathrm{s}$ Figure 1.

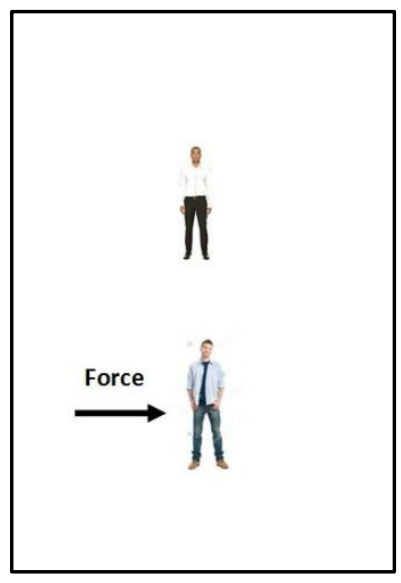

Figure 1. Initially, the two observers are in rest with respect to each other

Then, after stopping the force, he will be moving with a constant velocity of $60 \mathrm{~km} / \mathrm{s}$ in the $(x)$ direction with respect to obs(1) Figure 2.

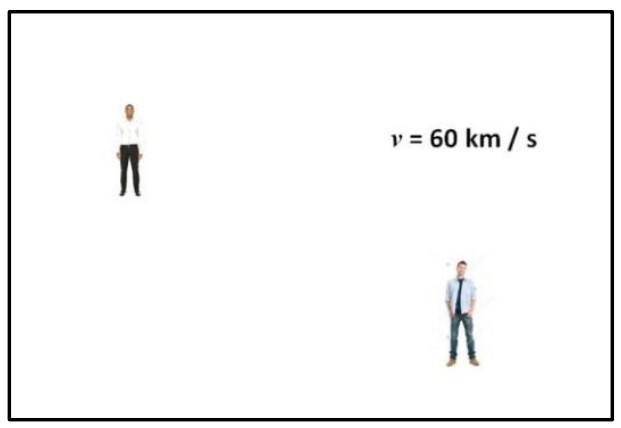

Figure2. observer 2 is moving with a constant speed with respect to observer 1

Since the space within this physical system is considered to be a three-dimensional classical space. The Galilean Transformation is hold within this system as an accurate one. Therefore, the spatial and time measurements between the two observers will be equal, and are given by: 


$$
\begin{gathered}
L=L^{\prime} \\
\Delta t=\Delta t^{\prime}
\end{gathered}
$$

Let us now move to the second phase of this experiment.

\section{2-2-Phase Two:}

In this step we will repeat the same previous experiment, with similar steps. But we shall make only one difference. We will fill the space with an omnipresent entity (non-mechanical medium), inside the same physical system. Then, we will try to investigate how the existence of this hypothetical medium will change this physical system. This hypothetical medium has two physical properties: The first property is that any of the two observes can trigger a propagating disturbance within the structure of this medium (wave), and we assume that this wave propagates at a speed of $(\mathrm{W}=100 \mathrm{~km} / \mathrm{s})$ with respect to the observer who is at rest state to this medium. The rest state is defined as that the speed between the observer and the medium is classically equals zero. Hence, a wave under this circumstance will propagate with the same speed (W) in all directions $(x, y, z)$, Figure 3.

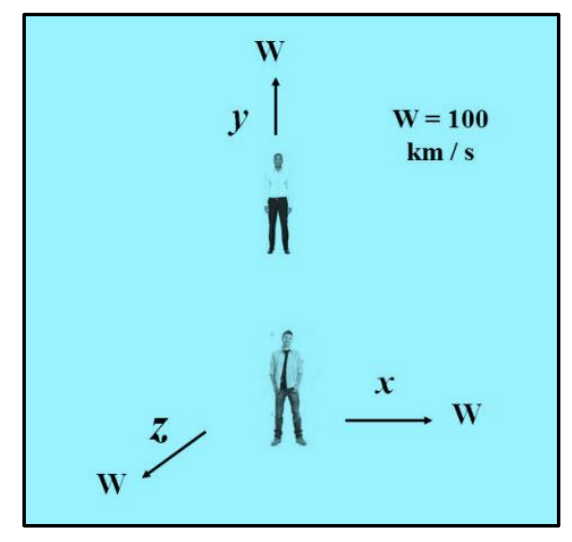

Figure3. Initially, both observers at rest with respect to each other, and with respect to the medium. Blue color indicates the existence of the medium.

The second physical property is that this medium is considered to have multiple equivalent rest states; it is multirest states medium. It simply indicates that this medium has more than one rest state. Therefore, any observer can leave or escape his rest state, and gets into another one. In other words, the ability of different observes to occupy different rest states within the same medium. These rest states are physically equivalent; physically, they are all the same. Metaphorically, this medium resembles a big mansion that has so many equivalent rooms ( rest states), and each inhabitant( observer) can leave his own room, and gets into another without any problem. Therefore, different inhabitants can occupy different rooms within the same mansion.

Now, we can repeat the same experiment that we have done in phase 1. Initially, the two observers will be considered at rest with respect to each other, and with respect to the medium Figure 3. As we have performed in the phase one, we shall apply a force - regardless of its magnitude - in $(x)$ direction to obs (2), for a specific time interval in order reach him a speed of $60 \mathrm{~km} / \mathrm{s}$. Then, after stopping the force, he will be moving with a constant velocity of $60 \mathrm{~km} / \mathrm{s}$ in the $(x)$ direction with respect to obs $(1)$.

The question now, if there is more than one rest state within this medium, how can we detect these multiple rest states? From obs (1) perspective, it cannot be the rest state that he currently occupy, and "was" occupied by obs (2) before his acceleration, therefore, let us name it rest state A, because it is his own rest state Figure 3 . Therefore, 
according to obs (1), if there are any more rest states exists within this medium, other than rest state A, which he currently occupy, it will only appear as constant motion with respect to him. This because any rest state represents an inertial state, and from obs (1) perspective, there are only two types of inertial states exist; the first, is his rest state (A), and the second state of inertia is constant motion with respect to him. From here, since obs (2) is now moving with a constant speed of $60 \mathrm{~km} / \mathrm{s}$ with respect to obs(1), he now occupies a rest state with respect to the medium other than the rest state (A), which is currently occupied by obs(1). So, let us call it rest state (B). Then, there is a question : how can obs(2) experimentally confirm that he now occupies a rest state with respect to the medium?

If we recall that this medium has another property beside its multiplicity of its rest states, which is that any observer can trigger a propagating disturbance within its internal structure, and this disturbance propagates at speed of $100 \mathrm{~km} / \mathrm{s}$ to any observer that is at rest with respect to the medium Figure 3. Hence, obs (2), can simply trigger a wave through the medium, and measures its speed in the three spatial directions $(x, y, z)$. If he is truly in a rest state with respect to the medium, he will measure the same speed of the propagating disturbance in all the spatial directions $(x, y, z)$, Figure 4 .

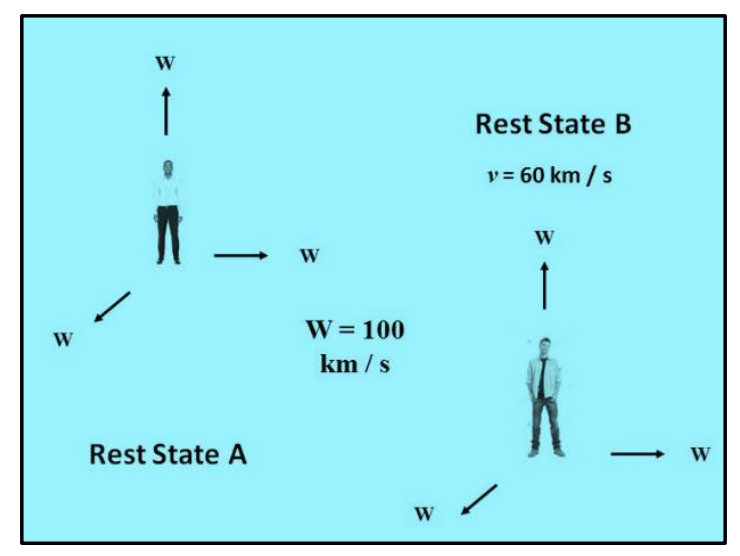

Figure 4. Both observers occupy a rest state with respect to the medium.

Now, let us draw the conclusions from this experiment:

1- A propagating disturbance in this medium will always travels with the same speed $\mathrm{W}=100 \mathrm{k} \mathrm{m} / \mathrm{s}$, regardless of the observes motion with respect to each other. Hence, this speed (W) will turn into a physical constant within this physical system.

2- Since rest state (A) and rest state (B) are physically equivalent, there will be no experiment that can differentiate between them. Hence, we can conclude that the laws of physics will always be the same in any rest state - or inertial frame.

3- By using a simple mathematical analysis based on the two conclusions above, we can say that both space and time measurements between the two observers will be " curved " by:

$$
\begin{aligned}
& \Delta t^{\prime}=\frac{\Delta t}{\sqrt{1-v^{2} / W^{2}}} \\
& L^{\prime}=L \sqrt{1-v^{2} / W^{2}}
\end{aligned}
$$


4- The previous conclusion implicates that the speed of this medium (W) will be the ultimate speed within this physical system. No observer cam move faster that $100 \mathbf{~ k m} / \mathrm{s}$ with respect to another observer.

Now, let us draw our attention to the third conclusion, which I considered the most important conclusion in this experiment, regarding our subject in this paper; emergence of the spacetime. Because, if you recall that regarding the physical system in which our experiment is performed, we have only made one change between phase 1 and phase 2, which is the addition of the omnipresent multi-rest states medium in phase 2. Due to this only one change, the nature of space has changed from the classical three-dimensional space ( phase 1 ) to a four- dimensional spacetime ( phase 2 ) in which the speed of the medium's wave $(\mathbf{1 0 0} \mathbf{~ k m} / \mathbf{s}$ ) has become a physical constant that governs the behavior of this spacetime. Intuitively, this indicates a strong relation between the medium and this new (emergent) spacetime in phase 2. Physically, it can be understood as the following: the multiplicity of rest state in our medium represents a physical property that enables this medium to conserve a constant speed of its propagating disturbances (waves), which is $\mathrm{W}=100 \mathrm{~km} / \mathrm{s}$, regardless of the observers movement with respect to each other, Figure 4. Motion or speed as a scalar quantity represents a spatial interval, divided by a time interval. Hence, in order for this medium to provide a constant speed of its propagating wave (W=100 km/s) for the all observers, it has to curve the spatial and time measurements with the same magnitudes that keep the speed of its propagating wave (W) unchanged for all observers. This relation becomes more obvious if we keep in mind that the speed ( W) at which this wave propagates depends mainly on the internal properties of its carrier medium, just as we have mentioned in the introduction, therefore, these properties represent the main factor which determines the value at which space and time curve with motion. But with more accuracy, what does it mean that this medium curves space and time measurements with motion?

The answer to this question can be understood from two opposite, but physically equivalent perspectives. The first one and regarding length. Consider same experiment that was performed in phase 2 . When obs(2) speed in $x$ direction changes from zero to $v=60 \mathrm{~km} / \mathrm{s}$, with respect to obs (1). The medium curves (changes) the value from $L$ to $L^{\prime}$. And since the value of $L^{\prime}$ is shorter than $L$ by the magnitude :

$$
\Delta L=L-L^{\prime}
$$

The curving process means that the medium is vanishing a length of value $(\Delta L)$, with respect to observer (1) along the $x$ direction.

But from an opposite perspective, when obs (2) speed in the $x$ direction changes from $v=60 \mathrm{~km} / \mathrm{s}$ to zero, with respect to obs (1), the medium curves (changes) the value from $L^{\prime}$ to $L$. And since the value of $L$ is greater (longer) than $L^{\prime}$ by a magnitude of :

$$
\Delta L=L-L^{\prime}
$$

The curving process means that medium is generating a length of value $(\Delta L)$, with respect to observer (1) inside his own frame of reference (his own rest frame A).

The key point now, when the value of $v$ approaches $\mathrm{W}$, we see that the value of $\Delta L$ approaches $\mathrm{L}$. Or when $v \approx w$, this yields $\Delta L \approx L$. From here when obs(2) speed in the $x$ direction changes from $v \approx w$ to zero with respect to obs (1), the medium curves (changes) the value from (zero $\left.\approx L^{\prime}\right)$ to $L$. And since the value of $L$ is greater (longer) than $L^{\prime}$ by the magnitude :

$$
\begin{gathered}
L^{\prime} \approx \text { zero } \\
\Delta L=L-L^{\prime} \approx L
\end{gathered}
$$


We see that the medium now is generating the whole value of $(L)$, to observer (1) inside his own frame of reference (his own rest frame $A$ ). Since $L$ stands for any arbitrary spatial value within the $x$ axis, and this experiment can be performed identically along the $y$ and $z$ axes. I assume we can reach to following result: regarding a specific observer within his own rest frame (or a frame reference), a muti-rest states medium generates the space along the $\mathrm{x}, \mathrm{y}$, and $\mathrm{z}$ axes.

Objectively, by considering the flow of time, its value can be expressed as a ratio ( $I$ ) between $\operatorname{Obs}(1)$, and $\operatorname{Obs}(2)$ as $I=\Delta t / \Delta t^{\prime}$. Hence, when the two observers are located in the same rest frame (A), time flows at a normal value between them $\Delta t=\Delta t^{\prime}, I=1$. But when obs(2) is moving with a speed of $v \approx w$ with respect to obs(1), the value of $I$ approaches zero which approximately means that time has stopped (cease to exist), with respect to this observer.

Now, if we repeat the same analysis that we have used for $(L)$ when obs(2) speed in $x$ direction changes from zero to $v=60 \mathrm{~km} / \mathrm{s}$, with respect to obs (1). The medium curves (changes) the value at which time flows from $I=1$ to $I^{\prime}=\Delta t / \Delta t^{\prime}$. And since the value of $I^{\prime}$ is lesser (slower) than $I$ by the magnitude :

$$
\Delta I=I-I^{\prime}
$$

The curving process of time means that the medium is diminishing (slowing) the of time by a value of a $(\Delta I)$, with respect to obs (1).

Oppositely, when obs (2) speed in the $x$ direction changes from $v$ to zero with respect to obs (1). The medium curves (changes) the value at which time flows from $I^{\prime}$ to $I$. And since the value of $I$ is greater than $I^{\prime}$ by the magnitude :

$$
\Delta I=I-I^{\prime}
$$

We see that that medium is generating (increasing) the value at which time flows by a magnitude of $(\Delta I)$, with respect to obs (1) inside his own frame of reference (his own rest frame A) . Completing the analysis, considering that $v \approx w$, just as we have done before with the length $(L)$, will lead us to the following conclusion. The medium generates the value at which time flows( $I$ ), with regard to observer (1) inside his own frame of reference (his own rest frame A).The main conclusion from this experiment is that both space and time flow (spacetime) do not represent the ultimate aspect of the nature; But spacetime is an emergent structure that results from the existence of a more complicated physical system, which is multi-rest states medium.

Concerning the problem of the EM waves propagation, we see that the assumption that we need to change about the characteristic of its carrier medium (ether) is that it should be defined as medium that has a multiple, equivalent rest frame. We simply replace the speed ( $\mathrm{W}=100 \mathrm{~km} / \mathrm{s}$ ) from the previous experiment, with the speed of EM waves ( $c=10^{8} \times 3 \mathrm{~m} / \mathrm{s}$ ). This version of ether was introduced in 2015 [20], and I choose to call it Sama Aether. The reason why I have chosen Sama as name for this version is that this word can be interpreted in many ways depending on the language which is chosen. Ultimately, these different interpretations share the same meaning. For example, in English language, this word can be derived from SAMeness Aether. It indicates the ether will be the same for any observer, regardless of the rest state that he (she) occupies for it.

If we get back to our problem, regarding the relationship between the ether and spacetime. We find that it does not just end there. This is because the spacetime represents the background of our physical interactions; all the interactions of nature occur there. To elaborate more, let us take two examples:

- Atmospheric air represents the carrier medium for sound waves. The relation between this carrier medium (air ), and our physical background( spacetime) is that air just occupies(exist within) this background apart from its gravitational effects. 
- Spacetime is the carrier medium for the gravitational waves. The relation between this carrier medium , and our physical background is that they represents the same thing.

- $\quad$ Regarding ether as a carrier medium for the EM waves, the situation is more complicated than that. If you recall that in phase 2 from our previous mind experiment, at the beginning of this phase, we assumed that the hypothetical multi-rest states medium exists within the space, and this gives the ability for both observers to excite it and therefore, trigger a propagating disturbance that moves with a speed of $\mathrm{W}=100$ $\mathrm{km} / \mathrm{s}$. Similarly, regarding the ether, we are have to assume that the ether occupy or exist within the spacetime. This can be understood, if you recall an electric charge emits EM when it gets acceleration. Therefore, when this charge accelerates, its acceleration causes an excitation (disturbance) in the structure of ether which propagates as wave(EM wave). The key point here is that this charge will not be able to disturb the ether unless, the ether exists in the space (our physical background); you cannot disturb( interact with) something that does not exist there at the first place. From here, we are have to say that the ether occupies (exists within) the spaceime. But on the other hand, when these waves propagates, the multiplicity of the rest states in this medium implies that these waves propagates at a constant speed for all observers, regardless of their motion with respect to each other. And from the previous experiment, we have seen that this leads to the conclusion that ether causes the emergence of the spacetime. Hence, we can put the two conclusions that we have reached, regarding the relation between the ether and spacetime in a more elegant statement; Ether exists within the spacetime, and its presence there causes the emergence of the spacetime itself. Another aspect of the relationship between the spacetime and the ether that should be considered is that if we accept the discreteness of the spacetime at the microscopic scale this implies the following : from our previous experience with modern physics, we have learned that discreteness usually results from underlying dynamical quantum process. Therefore, since the spacetime is just a reflection for the ether, then its discreteness intuitively means some sort of quantum behavior for the structure of ether at that microscopic scale.

\section{3-The Luminiferous ether moulds our universe:}

Energy is a scalar physical quantity that does not have a clear definition. But one of things that we know for sure about energy is that it comes in many different forms (types), like kinetic energy, and nuclear energy. At least, that how we usually visualize it. These forms can transform from type to another, hence, they are deeply connected in nature. But how many are they? Take a cup of tea for example. The internal (or thermal) energy is the first type of energy that we can initially observe. But if we probe this system down to its molecular level, the internal (or thermal) energy will just simply disappear during the probing process, and it will be replaced by the kinetic and potential energies of the tea's individual molecules, when we reach that molecular level. What we mean by "disappear" is to disappear as concept or type of energy, but not as a value, energy after all is conserved quantity. From this example, we can see that internal energy is not an independent or genuine from of energy. Its presence depends on the existence of other " more deeper forms" which are the kinetic and potential energies. As a physical concept, internal energy is neither kinetic energy nor potential energy, but its concept originates from both at the macroscopic scale. From here, we can call an emergent form of energy.

This can lead us to conclude the following; just like matter has elementary particle which their presence causes the emergence of more complex structures like molecules, and visible matter. Energy has elementary type(s) too, which their (its) presence within a physical system cause the emergence of other forms. These emergent forms can be visualized as forms of energy that do not have independent existence in nature, by their own and their presence within a physical system depend on other underlying forms (constituent forms). Examples will be considered as we proceed through our discussion. 
On the other hand the elementary form of energy can be seen as independent form which its existence does not depend on any underlying ( deeper) energy form. It resembles the concept of elementary particle, which can be defined as a particle that its existence does not depend on any underlying constituent particles. Kinetic energy is a good example for the elementary form. But surely, since energy can transform from one form into another, this entails that energy can transform between the elementary and emergent forms. Take for example; an electron interacting with a charged particle; its energy can transform from electric potential energy (emergent) to kinetic energy (elementary).

At first, this kind of analysis that classifies the types of energy into emergent and elementary might seem useless, and creates a state of unnecessary confusion. On the macroscopic level, I agree with this. But the important of this perspective comes at the microscopic level: when you probe the universe down to its basic level, all the emergent types of energy will simply disappear during the probing process, and you will end up with elementary type(s) only. At this basic microscopic level, any phenomenon that possess or require energy will be constrained by this(ese) type(s); It will be enforced to utilize the available elementary type(s) that exist at that basic level as an only option.

From here, we can rewrite the previous question regarding the number of energy forms in more accurate words as ; what are the elementary forms that do really exist in nature, and how many are they? The importance of this question comes from two points. The first is that this(ese) elementary type(s) will play a key role in shaping the universe at the microscopic scale ( basic level). The second point is that the answer to this question may pave the road to understand the nature of energy in a better way. But the answer to this question might not be easy, especially, if we keep in mind that the current cosmological observations point toward the existence of some sort of massive, mysterious entity; dark energy, which causes the universe to expand that its nature and origin are still obscure [21]. But despite of this, I can give a partial answer to this question by saying that within the ether as a physical system, there is only one type of elementary energy does exist, which is the kinetic energy. From here, the second postulate of our theory originates:

\section{The kinetic energy and potential energy are the only forms of energy for the ether's waves.}

Classical physics defines a wave as a propagating disturbance, which carry energy from one place to another. As we have discussed, at the basic microscopic level, only the elementary form(s) of energy do(es) exist(s). Therefore, ether's waves will be constrained or enforced to carry their energy in this(ese) available form(s) ; it is the only option that exists at that basic level. Now, with the existence of only one elementary form of energy in ether which is kinetic energy as we have earlier postulated. Ether waves will be constrained to carry their energy in this form. It represents a mechanical form of energy that is always carried on a particle (quantum), that has a linear momentum and acquire energy from motion. As we will see in the following discussion, there are different types of ether waves - not only EM field waves. Hence, the presence of all these different types of waves within the same universe (ether), shall open the possibility for them to interact with each other. These interactions will cause them to acquire an additional form of energy (emergent form), which is the potential energy. This explains the postulate above.

In the $19^{\text {th }}$ century, when the EM waves was detected for the first time. Classical physicists knew that these waves carry energy. The electric (magnetic) field represents a vector quantity that spread continuously in the space. Classical physicists also knew that energy comes in different forms. But this concept was not fully understood for them at that time. Hence, they simply assumed that electric (magnetic) field has its own form of energy in nature, which we can name it, the Electric Field Energy - just for the sake of description. This form of energy was assumed to spread continuously in space with electric field itself. But if their assumption was true, this meant that when these fields propagates as EM wave, its energy would spread continuously along the wave front; it would be conjoined with continuous spreading of the EM fields that make the wave. Experimentally, this would be interpreted by a continuous detection of the EM radiation, by any detector. Unexpectedly, this was not occurred, and it contradicted many phenomena, especially the photoelectric phenomenon. Hence, we can conclude that the assumption that was made by classical physicists was simply wrong. EM fields don not have a form of energy for their own, and they 
carry their energy in the kinetic form, although the fields themselves spread continuously in the space. This by itself enlightens us how the number of the elementary types of energy in nature is severely limited.

For more elaboration, let us visualize two electrons interacting with each other, by using the same perspective. A repulsive force will act on both electrons cause them to move (accelerate) away from each other. This movement is combined with kinetic energy for each electron. But where does it come from? The answer is that it comes from the electric field of the other electron, regarding each one of them. And these fields do not have an elementary form of energy of their own. Hence, they are constrained to carry their energy in a kinetic form (EM quanta). Hence, we can visualize their interaction as follows: each electron absorbs a kinetic energy (momentum) from EM quantum of the other electron's field. In turn, this absorbed energy(momentum) causes each electron to move away from each other, this by itself represents the act of EM force $(F)$. A strong action of this force, means more kinetic energy that each electron receives from the other. And since the strength of this force decreases as you move away $(r)$ from the electron. This implies that the kinetic energy of the field should decrease too ; $F=f(r)$. Therefore, in order to avoid this exhausting description, we can simply invent a concept of energy $(U)$ in order to simplify our description regarding this interaction. Approximately, this concept will be related to the previous force by $U=\int_{r_{1}}^{r_{2}} F d r$. This is simply the electric potential energy. From here, we can see that the electric potential energy - as a concept or form of energy - does not have a genuine existence in nature by its own. It can be seen as an emergent concept of energy that results from the spatial effects of the Field's kinetic energy originated from a charged particle; it presence as $a$ physical concept depends on the spatial existence of the kinetic energy (EM quanta) originated from a charged particle during the EM interaction. Therefore, the kinetic energy of EM quanta represents its constituent form, which by itself explains why the EM interactions do not occur immediately. But happen at a specific speed of $(c)$, which is assumed to be the speed of the EM quanta that carry the interaction. Although, we have reached this notion of EM interactions from classical concepts, it similar to the current version that we use today [22].

If we go back to our postulate, which states that the kinetic energy and potential energy are the only forms of energy for the ether's waves. The wave theory in classical physics does not forbid a wave from carrying its energy in the kinetic, and the potential forms, just like sound waves or all other mechanical waves. But a major difference between the EM waves and mechanical waves is the amplitude of the wave. In mechanical waves just like the sound wave, there are two velocity vectors Figure 5 . The first is associated with the amplitude of the wave, and the second is the propagation velocity vector. The particles which carry the energy of the sound waves move according to the amplitude's velocity vector. This is by itself results in a roughly continuous distribution of energy along the wave front. On the other hand, the amplitude in the EM waves - or generally, all ether waves - is just a field that spreads continuously in space, and is not related to a mechanical entity; it lacks motion in comparison with sound (mechanical) waves. The EM wave has only one type of movement, which points toward the direction of wave propagation. Therefore, the quanta (particles) which carry the wave's energy will have only one possible direction of movement, which is the direction of wave's propagation. Hence, light propagates as a wave that spreads continuously in space, due to the continuity of its constituent fields. But if we try to detect its energy experimentally, it will be detected in discrete, localized spots (quanta), and not continuously along the wave front. From this aspect, the radiation of EM waves can be visualized as a beam of quanta that move with the same direction of wave propagation.

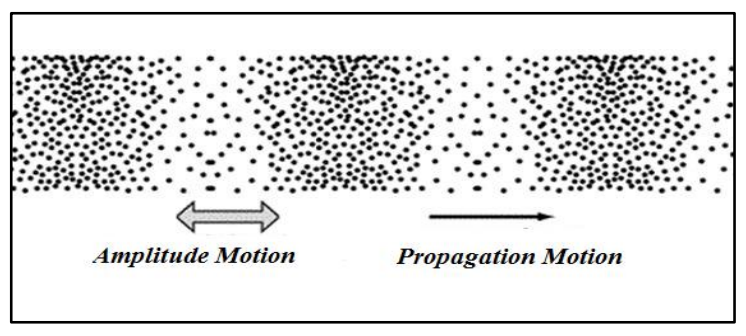


Figure 5. Sound waves in air. There are two types of motions in any mechanical wave. The amplitude motion and the propagation motion.

As a consequence of this, I think we can reach a simple answer to one of the most tantalizing questions in physics; what is light? light can be defined as a wave that carries kinetic energy; it seems that there will be no need to assume that light has a dual nature. Because, the wave theory by itself does not forbid a wave from carrying its energy in the kinetic form, just like saying sound is a wave that carries kinetic energy. By using a simple mathematical calculations based on the classical electrodynamics [20], we can conclude that each EM quantum should have an amount of kinetic energy equals to $\omega u$ [20]. Where $(\omega)$ is defined as the angular frequency of the wave, and $(u)$ is defined as a constant value that is dimensionally equivalent to Planck's reduced constant. But the reason why we define the energy of this quantum $K E=\omega u=p c$ as kinetic is going be considered later in this text.

As we have mentioned, classical physics defines a wave as a propagating disturbance, which carry energy from one place to another. But what type or form of energy does classical physics imply or impose to be carried by that wave? The answer is simply no; it does not impose any specific type or form to be carried by that wave. And from our previous discussion, we can see that this point can be determined by two things; first, the actual types (or number) of the elementary forms of energy that exist in nature. Secondly, at which level this wave occurs. If we take the basic microscopic level of the universe, any wave propagates at this level will be constrained - it can only carry - by the available elementary type that exist there, just like EM waves. But if we shift to a large macroscopic level, then any wave can utilize emergent forms. Take for example the string wave; its energy represents a combination between the kinetic and the elastic potential energy. The latter energy form is a collective addition of the EM potential energies of its constituent molecules (atoms), which are affected by elastic deformation. As we have discussed, electric potential energy is by itself an emergent form. Therefore, the elastic potential energy is an emergent form because its constituent forms are emergent (electric potential energy). This situation just resembles a composite particle (ice cube), which its constituent particle are also composite ( water molecule).

By shifting to a relevant point, as we have discussed, the EM wave is a disturbance that propagates through the ether, where (E, B) are the values of the amplitude for this disturbance. But, let us assume that there is another disturbance that propagates through the ether as a wave, but with different instantaneous amplitude value (S), where $(\mathrm{S})$ is the magnitude of a hypothetical field. The EM wave and the (S) wave share the fact that they are both propagating disturbances in the same medium (ether). But each one has a different amplitude value. Since (S) is a wave in ether, the previous postulate of ether, which states that ; the kinetic energy and potential energy are the only forms of energy for the ether's waves, should be applied for it, just like EM waves. This means that its energy will be carried on quanta, and since the wave of $(\mathrm{S})$ is a field wave, the quanta will be moving according to the direction of wave propagation, just as described earlier for light.

A difference will be made here, the quantum of the wave $(S)$ will be considered to have a detectable rest mass. Initially, this is a clear violation to the previous postulate, since the rest mass of this quantum has an equivalent value of rest energy $\left(E=m c^{2}\right)$, which is neither kinetic nor potential energy, and it will contribute to the wave's energy. This contradiction will be considered later. But for now, let us ignore it. If we consider a hypothetical experiment, which is the double-slit experiment for the (S) wave. A monochromatic radiation of $(\mathrm{S})$ is emitted from a hypothetical source, and passes through the doublesilt barrier to be observed latter by a detector screen. After a while of commencing the experiment, an 
interference pattern is expected to occur on the detector screen Figure 6. The source emits the (S) wave. The quanta which carry the wave's energy have a detectable rest mass, therefore, these quanta move with a speed less than that of light. Now, when the quanta hit the detector screen, it will be detected as localized, discrete spots.

Just like EM field, the energy of this wave is associated with field value. Therefore, on the detector screen, fringes of constructive interference are expected to receive the maximum amount of energy with time. Because the field reaches its maximum value at these areas. On the other hand, fringes of destructive interference are expected to receive the minimum amount of energy with time, due to a decreased value of the field there. Hence, on the detector screen a maximum intensity means a maximum number of quanta. Also, a minimum intensity, means a minimum number of quanta.

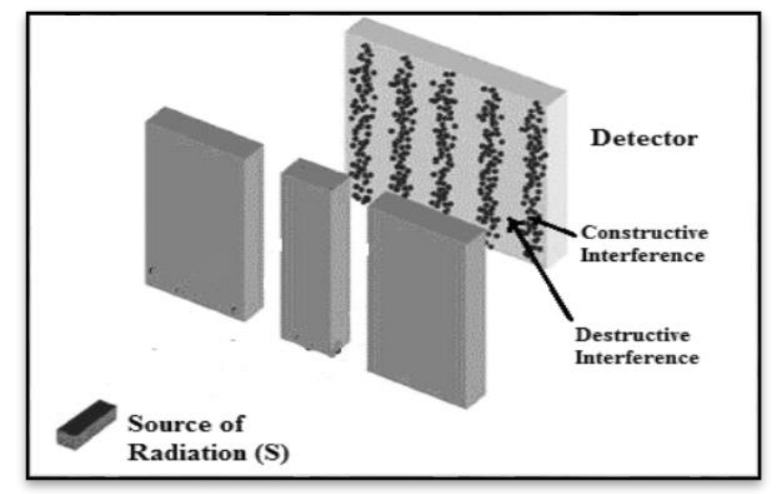

Figure 6 Interference pattern of $(S)$ wave on the detector screen

The conclusion that can be obtained from this experiment is that it illustrates the possibility to describe (S) waves by a completely different manner. Instead of using the instantaneous value of field (S) to describe the wave, another value can be used which is the wave function $(\psi)$. The meaning of this description is that the likelihood of detecting the quantum of the field (S) in a specific place at a specific moment is related to $|\psi|^{2}$ value there at the same moment. Therefore it represents a probability wave for the quantum of field (S). With a bigger picture, and by including more fields other than EM and the hypothetical (S), which all these field waves are propagating disturbances in the same medium, and the value of $(\psi)$ can be used to describe their waves instead of the value of their own fields. I think we can reach the following conclusion we can reach to the following conclusion; different field waves with different physical properties will be detected as different quanta (particles) with different physical properties.This description is identical to the concept of matter in modern physics. Therefore, will be no need to assume the existence of a dual behavior in nature; wave - particle duality. What do really exist are ether waves that share the same medium, but differ in their amplitude value. These field waves carry mechanical energy, which appear to us as probability waves for different elementary particles. The question that I think it should be asked now; how do we know that all these field waves are propagating disturbances in the same medium? How can we validate this hypothesis experimentally?

To answer this question, let consider the following example. The figure below shows a rope (string) which is attached to a tree. The boy in the figure is oscillating the rope (string) to produce a wave. 


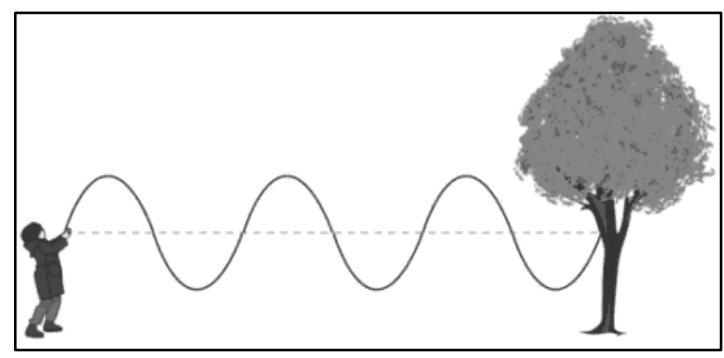

Figure 7. A rope wave

We know that wave is defined as a propagating disturbance. The medium represents the entity which is disturbed. From here, in the example above, the energy which associates the disturbance ( rope movement), and the wave's energy are the physical same thing:

The energy of the rope wave $=$ The energy of the disturbed medium( rope)

Also, the frequency of the rope wave is the number at which the medium (rope) oscillates per unit time:

$$
\text { The frequency of the rope wave }=\text { The frequency of the disturbed medium( rope) }
$$

Now, just hold this concept in your mind and shift to the ether. If EM wave is really a disturbance in a specific medium, then an identical concept should be hold. Therefore, when the ether is disturbed, these disturbances propagate from one place to another as waves, carrying energy with it. These disturbances will just appear to us as EM waves. From here, the energy of these propagating disturbances, and the energy of the EM waves are the same physical value, just like the example above:

\section{The energy of the EM waves = The energy of the disturbed ether}

Also, the frequency of the EM wave is the number at which the ether oscillates per unit time:

$$
\text { The frequency of the EM waves = The frequency of the disturbed ether }
$$

But what is interesting regarding the ether as a medium is that there is a constant ratio between these two values, as:

$$
\frac{\text { The energy of the disturbed ether }}{\text { The frequency of the disturbed ether }}=\text { constant value }(h)
$$

This constant value $(h)$ is Planck's constant. As explained earlier, the energy in ether waves is mechanical, which means it is carried on quantum (particle). Therefore, the energy in the mathematical expression above is the energy of the quantum itself ( $E$ ) that can be seen from $E=h v \rightarrow h=\frac{E}{v}$. Hence, if the Gluonic field wave (Gluon), the EM field waves, and all other field waves are really propagating disturbances in the same medium. Then they will be governed by the same mathematical expression above, with the same constant $(h)$. This is because the equation above relates the energy of the ether disturbance, with the frequency of that disturbance, regardless of the amplitude's value which is concerned. Whether it is an EM field, Electronic field, or any other field.

Concerning elementary particles in physics, Albert Einstein was the first to use the relationship $h=\frac{E}{v}$ which relates the energy of EM quantum to its frequency, regarding his work on the photoelectric effect [23]. Later on, the 
equation was generalized to include all the elementary particles in physics, when Louis de Broglie had hypothesized his matter waves. His idea of matter wave was just a hypothesis that had been inspired by the electron behavior in the hydrogen atom. Most likely he knew that expanding this equation to include different elementary particles should reflect a kind of similarity or symmetry between them with respect to their true nature. Since these elementary particles have different physical properties, but yet, they should share the same mathematical relation above. Certainly, it has reflected some kind of a common origin between them. But what is it? A simple justification is given in our ether theory. All the elementary particles are field waves in ether, which appear to us as probability waves for different quanta.

Kinetic energy is considered as an elementary form of energy. When a force of value $(\mathbf{F})$ is applied on a particle with a rest mass $\left(m_{0}\right)$, the kinetic energy $(K E)$ will represent the work $(W)$ which was done by this force, as $K E=$ $W=\int_{0}^{\vec{s}} \vec{F} . d \vec{s}=\int_{0}^{p} v d p \quad$ where (s) is the displacement acted upon the force $(\mathbf{F})$. Let us recall the relativistic equation of a linear momentum which is $\vec{p}=m \vec{v}=\frac{m_{0} \vec{v}}{\sqrt{1-v^{2} / c^{2}}}$. When this formula is applied in the equation of the kinetic energy above, this gives:

$$
K E=\frac{m_{0} c^{2}}{\sqrt{1-v^{2} / c^{2}}}-m_{0} c^{2} \approx \frac{1}{2} m_{o} v^{2}
$$

By defining the total energy $(E)$ of the particle as the sum of the rest energy $\left(E_{0}\right)$ and kinetic energy:

$$
E=K E+E \text { 。 }
$$

And by combining (1) and (2), another equation is obtained which is $\left(K E+E_{\circ}\right)^{2}=E^{2}=(p c)^{2}+E_{\circ}^{2}$. Now, when considering a massless particle, which lacks a rest mass, and therefore rest energy, the previous equation will give $K E^{2}=E^{2}=(p c)^{2}$, and by taking the root of this equation, this gives:

$$
K E=E= \pm p c
$$

Initially, this gives the possibility for the existence of positive and negative kinetic energy. By considering the positive value of this kinetic energy which appears in equation (3), you can note that it is different from the kinetic energy in (1) from two aspects:

- The kinetic energy in (3), is not a function in the particle's speed as does (1), because the particle is massless, and considered to move with a constant speed $(c)$. Therefore it cannot be defined as the work done by a specific force, in certain displacement just like the kinetic energy in equation (1).

- The kinetic energy in (3), represents the total energy of the particle, in comparison with the kinetic energy in (1) which represents only the additional part of energy which is acquired from motion( besides rest energy).

Regarding these two differences the kinetic energy in (3) it is not the same as that in (1). Therefore, the simplest way to deal with the kinetic energy in (3) is to consider it as another or second form $\left(K E_{2}\right)$ of kinetic energy. Therefore, the total energy for "a free" massless quantum ( just like the EM quantum) is completely kinetic $K E_{2}=$ $p c$. If we recall our previous postulate, which states that, the kinetic energy $(K E)$ and potential energy $(P E)$ are the only forms of energy for ether's waves, a problem emerges spontaneously. The potential represents the effect of the conservative forces which acting on the particle. Therefore, from this postulate, when a particle is free (no action of any force), the kinetic energy will be the only form of energy. Initially, this makes a problem because many ether's waves are associated with quanta that have a detectable rest mass (like electron), and we know from the equation of energy-mass equivalence that this rest mass has an equivalent amount of rest energy. Apparently, this rest energy is neither kinetic energy nor potential energy, and still it contributes to the wave's energy $\left(E=E_{o}+K E+P E\right)$, and 
from here comes the contradiction. Furthermore, even a massless particle (like EM quantum) may not be completely massless; it may contain for some reason, undetectable amount of rest mass(energy), which creates more contradiction with our ether concept. To solve this problem, if we hold the second postulate of ether as a valued principle, the rest energy $\left(E_{0}\right)$ should be considered as a form of kinetic energy or simply a third form of kinetic energy $\left(K E_{3}\right)$. Because at this situation, the total energy of any free particle will be completely kinetic $\left(E_{0}+K E=\right.$ $\left.K E_{3}+K E\right)$. The general notion or idea behind the kinetic energy is obtained from (1), which it represents an amount of energy that is acquired from motion. Therefore, redefining the rest energy $\left(E_{0}\right)$ as a kinetic energy $\left(E_{0} \equiv K E_{3}\right)$ is done by defining it as a function in some kind of motion or speed $\left(v_{0}\right)$ as $K E_{3}=f\left(v_{0}\right)$. From the equation of $K E_{1} \approx \frac{1}{2} m_{0} v^{2}$, we see that that the usual kinetic energy is directly proportional to $v^{2}$, hence, we can intuitively assume that:

$$
\begin{gathered}
E_{0} \equiv K E_{3} \\
K E_{3} \propto v_{0}^{2}
\end{gathered}
$$

Apparently. the main problem with this step is that the particle is at rest, which means it should not have any kind of motion. Therefore, what is this speed $\left(v_{0}\right)$, and where does it come from? To answer this question, let us review the principle that Einstein's GTR stands on; the Equivalence Principle. It can be stated as; all physical experiments conducted in a gravitational field and in an accelerated frame of reference give identical results. For explanation let us consider the following experiment: assume there are two observers, each one of them is located away by a distance of $\boldsymbol{k}$ from the surface of a big spherical and static planet with rest energy ( $\left.E_{\text {planet }}\right)$, Figure 8.

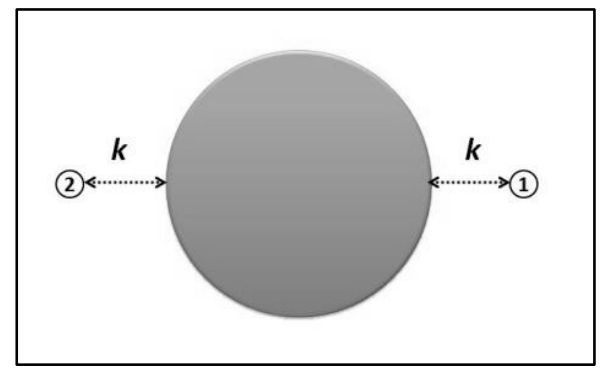

Figure 8, Two observers located away from the surface of a big, static planet - Scales in this picture are intentionally deviated from the experiment requirements for the sake of demonstration.

The two observers are located at opposite sites within the same spatial axis. Their measurements regarding space $(\boldsymbol{k})$ and time should be considered relatively very small, in order to ignore any kind of tidal effects, which can compromise the validity of the equivalence principle itself. Hence, the surface of the planet appears as a flat surface, as a sort of approximation, instead of convex. Regarding each observer, at first, he /she is located away from the surface by a small distance of $(\boldsymbol{k})$. After a while, each observer touches the surface of the planet due to its gravitational acceleration.

The key insight which can be obtained from the equivalence principle, regarding this experiment is that it implies that each observer can simply assume that he (she) was initially at rest, and the surface of the planet was accelerated toward him. Each observer, on his (her) own side of the planet can claim this. Therefore, if we combine the observations of the two observers, in a single statement, we can intuitively " assume" that the planet has accelerated in two opposite directions at the same time. Acceleration can be defined as change in velocity with respect to time. Hence, for an observation that lasts a period of time, you cannot have acceleration without motion, which entails that the planet has moved in two opposite direction at the same time. Let us call this type of motion $\left(v_{0}\right)$; rest motion [24-25]. It follows a very simple equation for a classical particle $\vec{v}_{0}+\left(-\vec{v}_{0}\right)$. 
A more detailed and accurate approach for this problem was considered previously [24], which entails that for a static, spherically symmetric planet with no electric charge, and a specific radius $\left(r_{0}\right)$, The kinetic energy is :

$$
K E_{3}\left(v_{0}\right)=r_{0} c^{2} v_{0}^{2} / 2 G
$$

Where $v_{\circ}$ is defined as the rest speed of the planet. It is mathematically but not physically equivalent to its gravitational escape velocity from its surface. One of the insights, regarding this concept of rest motion is that a black hole can be considered as an object moves with a rest speed equal to the speed of light $\left(v_{\circ}=c\right)$.

In physics we have different types of motion like linear and circular motions. It is important to note that this rest motion is not an additional type of motion. This is because motion can be defined as change in position, with respect to time $\vec{v} \neq 0$. But in the rest motion, the particle moves in two opposite spatial directions at the same time. Therefore, its positive and negative displacements cancel each other simultaneously. This in turn causes a change in the positions around the particle, but the particle's position remains unchanged; the particle is initially, and eventually at rest $\vec{v}=0$. Therefore, this motion can only be considered as a type of rest. Hence, we have two types of rest for a classical point particle:

- $\quad$ An absolute rest, where both $v=v_{\circ}=0$.

- A rest motion where $v=0, v_{\circ}>0$.

On the other hand, this does not affect our concept which defines the rest energy as kinetic energy. Because this energy is $f\left(v_{0}\right)$. Both linear motion $\vec{v}$, and this rest motion are dimensionally equivalent $v=v_{0}=$ [length] / [time].

We have concluded that the third form of kinetic energy $\left(K E_{3}\right)$ for a massive object like Earth, and its gravitational effects (spacetime curvature) are just two sides one coin [24]. This in turn illuminates the way to see that the type of energy which is stored within the gravitational field that can affect any object that falls in that field is just kinetic in origin. Eventually, I think this may lead us to ask ourselves; is kinetic energy the only elementary form of energy in nature? From our previous historical experience with the universe, I think we can learn that questions like this cannot be answered with a definite precision, but we can try.

\section{4- Ether's Prospects:}

One of the lessons that can be obtained from the historical development of physics is that its progress can appear as an outcome of a successful integration between the theoretical demands and observational clues. Today, I can see that both of them stand behind the ether. Theoretically, regarding our human knowledge, since its early beginning, nature has always been like some sort of a big disordered jigsaw puzzle. With the development of science, each time, it has provided us with separate pieces of that puzzle. This in turn, has created an urging quest to see the big portrait that these separate, disorganized pieces stand for. The quest to fulfill unification that is so deep which not only unify the physical interactions that we observe in nature. But also unify physics itself, and bring it into a single, straight epistemological context. That can remove the contradictory wall, which has divided it into classical (macroscopic), and modern (microscopic) concepts. From our previous discussion, we can see that the ether represents a fertile substrate to achieve that goal.

Observationally, in June 2020, Tom Zlosnik and Constantinos Skordis, two researches have published a manuscript (preprint) that contains what can be considered as key observational result, regarding the ether. Their model which is entitled Relativistic Modified Newtonian Dynamics, or RelMOND for short. It represents a continuation to the ether theory that was introduced in 2000, by the two theorists Ted Jacobson and David Mattingly[26]. In 2000, both theorists were able to combine Einstein's GTR with an ether model in one single 
mathematical framework - for the sake of honesty, I was not aware about their work until 2018 - They introduced the ether as a field rather than some sort a non-mechanical substance, but initially, at the cosmological scale it would not make a big difference. Because whether you consider the ether as a field or as kind of non-mechanical physical entity, both will act as a preferred frame of reference within a relativistic universe. A major goal was to see how the existence of this preferred frame (ether) is going to affect GTR, and what the consequences that would emerge from a scenario like this. In 2018, Tom Zlosnik and his co-workers, were able to deduce unexpected prediction regarding this scenario. They have reached to the conclusion that at the largest observable cosmological scale, this ether field can behave strictly like a kind of invisible matter, with strong gravitational effects that can hold the visible matter as some sort of glue. In other words, it can acts like what Physicists today call dark matter [27]. In the past June, Zlosnik and Skordis have published a manuscript (preprint) that contains an observational clue regarding this prediction[28]. In their ReLMOND, they show that the existence of this kind of ether field can accurately reproduce the temperature of the Cosmic Microwave Background (CMB). One of the key observations that has always been assumed that Lambda Cold Dark Matter(LCDM) is the only theory that can reproduce

this observation [29]. Moreover, in September 2020, I had a contact with Dr.Zlosnik, and he enlightened me that their calculations are in a good fit with polarization spectrum, and matter distribution. Therefore, ether is a promising candidate to solve one of the biggest cosmological mysteries.

\section{5 - Conclusion:}

We have deduced that the spacetime is an emergent structure that rises from the existence of multi-rest states medium, and the existence of this medium can ontologically justify the origin of wave-particle duality, and the existence of the equivalence principle.

\section{References:}

[1] Huggett, N et al. (2013) Emergen spacetime and empirical (in)coherence. Studies in History and Philosophy of Science Part $\mathrm{B}, \mathbf{4 4}$ ( 3 ).

[2] Martens, N. (2019) The metaphysics of emergent spacetime theories. Philosophy Compass, Vol 14, No 7.

[3] Seiberg, Nathan (2006). Emergent Spacetime.

[4] Rovelli, C. (2004) Quantum Gravity. Cambridge University Press, Cambridge.

[5] Huggett, N \& Wuthrich, C. (2020) The " emergence" of spactime in string theory.

[6] Isam, A. (2018) Spacetime Discreteness: Shedding Light on Two of the Simplest Observations in Physics. Journal of Modern Physics, 9.1415- 1431.

[7] Einstein, Albert (1961), Relativity: The Special and the General Theory, New York: Three Rivers Press (published 1995).

[8] Bohr, N. (1913) On the constitution of atoms and molecules. Philosophical Magazine, 26 ( 6).

[9] De Broglie, L. (1924) A Tentative theory of Light quanta. Philosophical magazine, 47.

[10] Weinberger, P. (2006) Revisiting Louis de Broglie's Famous 1924 paper in Philosophical Magazine. Philosophical

Magazine Letters, 86 (7).

[11] Beiser, A.(2003) Concepts of Modern Physics ,6th edition, McGraw Hill companies.

[12] Einstein, A\& Infeld, L(1938) .Evolution of Physics. Cambridge University Press, London. p 159.

[13] Wuensch, Daniela. (2003) The fifth dimension: Theodor Kaluza's ground-breaking idea. Annalen der Physik ,12 (9).

[14] Andre Morgado Patricio. (2013) 5D Kaulza-Klein theories - a brief review.

[15] Fishbane, P.M, Gasiorowicz, S.G, and Thornton, S.T."Physics for Scientists and Engineers with modern physics", Pearson Prentice Hall.

[16] Whittaker, E.T. (1910), 1. Edition: A History of the theories of aether and electricity, Dublin: Longman, Green and Co.

[17] Michelson, A. A. \& Morley, E. W. (1887), On the Relative Motion of the Earth and the Luminiferous Ether, American Journal of Science 34.

[18] Abbot, B.P. ; Abernathy, M.R; et al (2016) Observation of gravitational waves from a binary black hole merger. Physical Review Letters, 116 (6). 
[19] Georges, Alexandre. (2018) Incompatibility between Einstein's general relativity and Heisenberg's uncertainty principle. Physics Essay, 31(3).

[20] Isam, A (2015). A New Medium for Matter and Light Waves. American Research Journal Physics, 1 (1).

[21] Edmund J. Copeland, M ; et al. (2008) Dynamics of dark energy. International Journal of Modern Physics D, 15 (11).

[22] Martin, B. R. (2006) Nuclear and Particle Physics. John Wiley \& Sons, Ltd. West Sussex, England.

[23] Einstein, A. (1905) On a heuristic point of view concerning the production and transformation of light, Annalen der Physik .

[24] Isam, A. (2019) Gravity, where does it come from?

[25] Isam, A. (2016) The basic idea behind the relativistic and quantum concepts. Journal of Modern Physics, 7.

[26]Eling, Christopher \& Jacobson, Ted \& Mattingly, David (2004). Einstein-Aether Theory.

[27] Zlosnik,Tom \& Urban, Frederico; et al. (2018). Spacetime and dark matter from spontaneous breaking of Lorentz symmetry. Classical and Quantum Gravity, 35 (23).

[28] Skordis,Constantinos \& Zlosink, Tom.(2020) A New relativistic theory for modified Newtonian dynamics.

[29] Pardo, Kris \& Spergel, David. (2020) What is the price of abandoning dark matter? Cosmological constrains on alternative gravity theories. 